\title{
La congruencia entre los juicios de legitimidad de la institución pública, los medios y los ciudadanos
}

\section{The congruence between the legitimacy judgments of the public institution, the media and citizens}

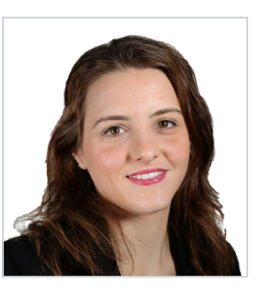

Recibido: 15/01/2020 - Aceptado: 30/04/2020

\section{Resumen:}

La investigación explora la dinámica de la legitimidad de un líder público y su organización con responsabilidades ejecutivas en un gobierno central. El objetivo de esta investigación es recuperar la confianza de los ciudadanos a las instituciones públicas mediante el bien intangible legitimidad. Por ello, se pretende analizar la congruencia que hay entre los juicios de legitimidad que sobre un ministerio de educación -Ministerio de Educación del Gobierno de España, con ministros José Ignacio Wert e Iñigo Méndez de Vigo (2011-2015)-formulan la propia organización, los medios y los ciudadanos. El diseño metodológico para la observación empírica es triangular y consiste en un análisis de contenido cuantitativo de los juicios de legitimidad, contrastando tres puntos de observación. En concreto, de la organización se analiza la comunicación en Twitter de los ministros y del ministerio, así como las notas de prensa y comunicados; de la prensa se analiza la prensa impresa y los perfiles de los diarios en Twitter; y de los ciudadanos, los tweets. Los resultados manifiestan cómo es la comunicación de una organización pública en su interacción con los diferentes actores, y cómo esta interacción puede afectar a su legitimidad y, en último término, a los ciudadanos.

\section{Palabras clave:}

Comunicación de instituciones públicas; bienes intangibles; sector público; legitimidad; confianza.

\section{Received: 15/01/2020 - Accepted: 30/04/2020}

\section{Abstract:}

This research explores the dynamics of legitimacy in a public leader and his organisation with executive responsibilities in a central government. The aim of this study is to rebuild the trust of citizens in public institutions by means of the intangible asset legitimacy. Thus, our purpose is to analyse the congruence between the legitimacy judgements which the own organisation, the media and citizens make about a ministry of education-Ministry of Education of the Government of Spain, with José Ignacio Wert and Iñigo Méndez de Vigo as ministers (2011-2015)-. The empirical observation follows a triangular methodological design and consists in a quantitative content analysis of the legitimacy judgements, contrasting three points ofobservation. Specifically, an analysis of the following has been carried out: as far as the organisation is concerned, the communication on Twitter by the ministers and the Ministry, as well as press releases and announcements; regarding the media, print media and newspapers profiles on Twitter and, as for citizens, their tweets. The results show the communicative characteristics of the interaction between a public institution and the different actors, as well as how this interaction may affect its legitimacy, and ultimately, citizens.

\section{Keywords:}

Communication by public institutions; intangible assets; public sector; legitimacy; trust.

\footnotetext{
Cómo citar este artículo:

Robles López, C. M. (2020). La congruencia entre los juicios de legitimidad de la institución pública, los medios y los ciudadanos. Doxa Comunicación, 30, pp. 351-368.
} 


\section{Introducción}

Recientes informes de organismos internacionales como el Banco Mundial o Naciones Unidas han revelado la necesidad de llevar a cabo investigaciones sobre la pérdida de confianza en el sector público. Dichos organismos han recomendado facilitar el acceso a la información, impulsar la participación, construir la legitimidad, etc. con el objetivo de acortar el distanciamiento entre las instituciones públicas y los ciudadanos.

Este trabajo se centra en los bienes intangibles, en concreto en la legitimidad, para dar respuesta a este problema. Se trata de un activo intangible basado en la percepción. No se puede crear desde cero porque se basa en experiencias reales, pero si se puede realizar una estrategia comunicativa para desarrollarlo.

La presente investigación explora la dinámica de la legitimidad de un líder público y su organización con responsabilidades ejecutivas en un gobierno central. Más específicamente, se analiza lo que está en la base para la construcción de este intangible: la congruencia que hay entre los juicios de legitimidad que la propia institución pública, los medios y los ciudadanos formulan sobre el ministerio y sus ministros.

El caso de estudio seleccionado ha sido los ministros de Educación, Cultura y Deporte en España de 2011 a 2015, José Ignacio Wert e Iñigo Méndez de Vigo y el Ministerio de Educación español. En aras de contextualizar la situación, el ministro José Ignacio Wert ocupó su cargo desde el 22 de diciembre de 2011 hasta el 24 de junio de 2015. Dicho ministro presentó el 31 de enero de 2012 un proyecto para reformar la educación de secundaria que originó una huelga por sindicatos, docentes y estudiantes. También tuvo desacuerdos con rectores, con la política educativa de Cataluña, etc. Por otro lado, también tomó decisiones controvertidas con la obtención de becas y el programa Erasmus. Tras los continuos desencuentros con la comunidad educativa, decidió dejar el cargo. Según los datos del Centro de Investigación Sociológica (CIS), José Ignacio Wert se encontraba entre los ministros con peor puntación.

El 25 de junio de 2015, Iñigo Méndez de Vigo tomó posesión como ministro de Educación, Cultura y Deporte.

El objetivo de este trabajo es acercar a los ciudadanos a las administraciones públicas para recuperar la confianza a través del bien intangible legitimidad. También se trata la legitimidad online y la legitimidad mediática como bienes intangibles.

\subsection{La legitimidad como bien intangible y su concepción online}

Los bienes intangibles están presentes en el sector público (Bossi et al., 2005) y son necesarios para satisfacer las exigencias de los ciudadanos en cuanto a transparencia y excelencia en los servicios (Sánchez, 2008).

A raíz de la crisis económica, las administraciones públicas están perdiendo la confianza de los ciudadanos. Por un lado, no pueden cubrir las necesidades de los ciudadanos (Thomas, 2013) y, por otro lado, no pueden conservar el nivel de excelencia (Luoma-aho, 2007). Este contexto también endurece los juicios que formulan los ciudadanos ante la corrupción (Uslaner, 2010; Canel y García-Molero, 2013; Chen et al., 2013).

El concepto de legitimidad ha sido abordado desde diferentes enfoques que parten de la definición de Suchman (1995). Este autor define la legitimidad como "una percepción generalizada de que las acciones de una organización son desea- 
bles, adecuadas o apropiadas dentro de un sistema socialmente construido con normas, valores, creencias y definiciones" (1995: 574).

Por otro lado, autores como DiMaggio y Powell (1991), Bitektine (2011) o Robles (2019) lo han relacionado con los juicios que formulan los públicos sobre las acciones que llevan a cabo las organizaciones.

En esta investigación abordamos la legitimidad como un bien intangible en el sector público tanto en la organización pública como en el líder que la dirige. La legitimidad cumple con los rasgos recogidos en la definición de activo intangible para el sector público de Canel y Luoma-Aho:

"Activo no monetario (sin sustancia física), que habilita y da acceso a activos tangibles y se activa a través de la comunicación, basada en eventos pasados (y vinculado al comportamiento de la organización); por lo tanto, da lugar a un recurso que es identificable y del cual se espera que fluya un beneficio/ valor (social, monetario, etc.) futuro (a largo plazo) tanto para la organización como para los stakeholders/ ciudadanos" (2019: 77).

La legitimidad cumple el primer rasgo puesto que es una percepción y no tiene forma física y es un bien no monetario. En segundo lugar, también se puede considerar un bien intangible porque está relacionado con el pasado de la organización. Los ciudadanos cuando formulan sus juicios de legitimidad se basan en experiencias y en informaciones que tienen con las administraciones públicas (Luoma-aho, 2007; Canel y Luoma-Aho, 2017; Robles, 2019).

En tercer lugar, la legitimidad también puede ser un bien intangible porque genera relaciones con los públicos de interés, es decir, incrementa la confianza de los ciudadanos. En cuarto lugar, los bienes intangibles en el sector privado generan recursos que conllevan un beneficio económico. Esta idea se puede llevar al sector público y concluir que la legitimidad ayuda a la supervivencia de las administraciones públicas.

Analizados todos estos rasgos, se puede afirmar que la legitimidad es un bien intangible en el sector público. Para las organizaciones públicas supone un beneficio tanto económico como social (Robles, 2017).

En cuanto al carácter online de la legitimidad, nace ante los cambios sociales. Los ciudadanos cada vez son más exigentes con las organizaciones y piden que sean legítimas y justifiquen cuestiones económicas, sociales y medioambientales. Estos cambios en las formas de relacionarse, lleva a las empresas hacia la legitimidad corporativa y hacia nuevas formas de diálogo (Colleoni, 2013: 229).

Catelló, Etter y Arup (2016) llevaron a cabo una investigación sobre legitimidad online en la que proponen una estrategia de medios sociales. Concluyen que las organizaciones pueden ganar legitimidad en los medios sociales si evitan las jerarquías a la hora de relacionarse con sus públicos de interés. También afirman la importancia de considerar que la sociedad está conectada a la red.

Las redes sociales han facilitado que la legitimidad surja de un proceso de reconocimiento y diálogo entre las organizaciones y sus stakeholders. El engagement no viene definido por la organización, las redes sociales posibilitan la participación de diferentes stakeholders.

En definitiva, se puede concluir que "legitimidad online, al igual que la legitimidad offline, sigue siendo un juicio respecto a las actuaciones de la organización” (Robles, 2017). Se trata de un juicio que se formula en base a unas normas sociales 
e independientemente de la información que emiten las administraciones públicas y los medios de comunicación en los medios sociales. Por consiguiente, las administraciones públicas tienen que realizar una gestión estratégica de la legitimidad online, al igual que de la legitimidad.

\subsection{Cuáles son los tipos de legitimidad}

Es importante explorar los diferentes tipos de legitimidad para analizar los elementos a los que se refieren los ciudadanos cuando elaboran sus juicios. La literatura propone tipologías de legitimidad para identificar los diferentes tipos de juicios emitidos respecto a las características observadas o dadas sobre una organización (Deephouse y Carter 2005; Díez et al. 2010; Bitektine 2011).

La clasificación más completa es la que sugiere Suchman (1995). Este autor identifica tres tipos de legitimidad: la pragmática, la moral y la cognitiva. Afirma que esta tipología se basa en que los juicios se formulan en relación con los estándares sociales, pero hay matices que diferencian el objeto del juicio.

En investigaciones como la de Canel (2015), Robles y Canel (2017) y Robles, (2019) se ha partido de la tipología de legitimidad moral de Schuman (1995) para analizar la comunicación de los bienes intangibles. Esta tipología es la siguiente:

- La legitimidad procedimental es la que se apoya en las evaluaciones que la gente hace sobre los procedimientos y procesos que las administraciones públicas han seguido para la producción de resultados.

- La legitimidad personal es la del liderazgo.

- La legitimidad consiguiente hace referencia al juicio de los resultados de la gestión pública.

- La legitimidad estructural tiene que ver con los edificios, recursos, políticas laborales, etc.

Las administraciones públicas pueden utilizar la investigación sobre los juicios de legitimidad, que formulan los ciudadanos, para gestionar la legitimidad y satisfacer sus expectativas y necesidades.

\subsection{La legitimidad en un sistema mediático híbrido}

En este epígrafe vamos a explorar el concepto legitimidad mediática y contextualizaremos el sistema mediático híbrido.

La legitimidad mediática es la legitimidad que se muestra en los medios de comunicación (Bitektine, 2011). Para hacer una aproximación al concepto de legitimidad mediática abordaremos a los autores Pollock y Rindova. Estos autores concluyen que "el rol de los medios de comunicación es ser intermediarios de la información" (2003: 631). En su investigación consideran que la forma en la que los medios emitan o no emitan la información también influye, las pueden hacer más deseables y más legítimas (2003: 631). Por consiguiente, los juicios que formulen los inversores pueden estar influenciados por cómo se presenta la información que emiten los medios de comunicación.

En este sentido, podemos afirmar que la legitimidad mediática está relacionada con el juicio que emiten los medios de comunicación sobre una administración pública. Los autores Robles y Rodríguez (2017: 56) concluyen que la legitimidad mediática es un intangible para la organización, pero que depende de las evaluaciones que realicen los medios de comunicación. Además, afirman que "los medios son generadores de opinión pública y catalizadores de la atención, y por 
este motivo, cualquier organización pública o privada debe vigilar el eco mediático y adoptar estrategias que velen por su legitimidad" (2017: 56).

Respecto al eco mediático, tal y como acuñó Chadwick (2013) nos encontramos en un sistema mediático hibrido, es decir, en un contexto en que encontramos medios tradicionales y medios sociales. Según este autor, ambos medios se encuentran en un mismo contexto, pero son los medios tradicionales los que marcan la agenda e influyen más en la sociedad.

No obstante, los medios tradicionales se están creando perfiles en los medios sociales para adaptarse a este contexto onli$n e$ (Chadwick, 2013). Por ejemplo, la red social Twitter se ha convertido en una herramienta profesional de comunicación (Ramos del Cano, 2013).

\subsection{Los medios sociales en las instituciones públicas: Twitter como herramienta de comunicación}

En este apartado nos adentraremos en el nuevo contexto mediático y en el uso que hacen las administraciones públicas y sus líderes políticos de los medios sociales.

Las organizaciones públicas tienden a utilizar los medios tradicionales para ganar visibilidad, pero los medios sociales han aparecido como un nuevo escenario (Baamonde, 2011).

Este nuevo contexto mediático ha impulsado nuevas formas públicas de participación, en las que los ciudadanos pueden interactuar con las administraciones públicas tanto generando contenido como marcando la agenda (Jenkins, 2006). Con las redes sociales está cambiando el modo en el que la ciudadanía participa en el debate social y político (Bennett \& Segerberg, 2014).

La aparición de los medios sociales potencia la participación de los ciudadanos en las políticas públicas y la bidireccionalidad (Bertot et al., 2010; Chun et al., 2010). Las instituciones públicas y sus líderes pueden conocer las necesidades y exigencias a través de los medios sociales. Según los autores, Congosto y Aragón "los ciudadanos pueden interactuar con ellos, hacerse eco de sus mensajes, seguir sus lemas, mostrar su desacuerdo o simplemente citarlos por su nombre real” (2012: 51).

Sin embargo, Giansante (2015) afirma que ni los partidos políticos ni los líderes que-dirigen instituciones públicas sacan el máximo rendimiento a los medios sociales. Las utilizan como un medio más para amplificar sus mensajes. En este sentido, entendemos que se puede extrapolar a las instituciones públicas porque también pueden interactuar con los ciudadanos a través de los medios sociales.

En el caso de las redes sociales, las administraciones públicas las utilizan cada vez más. Sin embargo, en la mayoría de los casos la información es unidireccional y no se fomenta ni la participación ni el diálogo con los ciudadanos (García, 2012; De Ramon, 2014; Manfredi y Femenía, 2016; Robles, 2019).

En los últimos años se han llevado a cabo investigaciones sobre la actividad de las administraciones en redes sociales, especialmente en Twitter (Romero y Mena, 2013; Vázquez, 2013; Martínez y Piñeiro, 2014; Pardo, 2014; Simelio y Molina, 2014, Catalina et. al, 2015). 
La red social Twitter es considerada un medio social que facilita el acceso a la información y la interacción con las administraciones públicas (Grimmelikhuijsen \& Meijer, 2014).

\section{Metodología}

La metodología empleada en esta investigación es triangular, pues se pretende analizar la congruencia de los juicios de legitimidad que se realizan sobre el Ministerio de Educación, Cultura y Deporte entre la propia organización, los medios y los ciudadanos.

Se estudia cómo interactúan los mensajes del ministerio y ministro, en concreto Ministerio de Educación del Gobierno de España, con ministros José Ignacio Wert e Iñigo Méndez de Vigo (2011-2015), con los medios y ciudadanos.

Imagen 1. Diseño metodológico

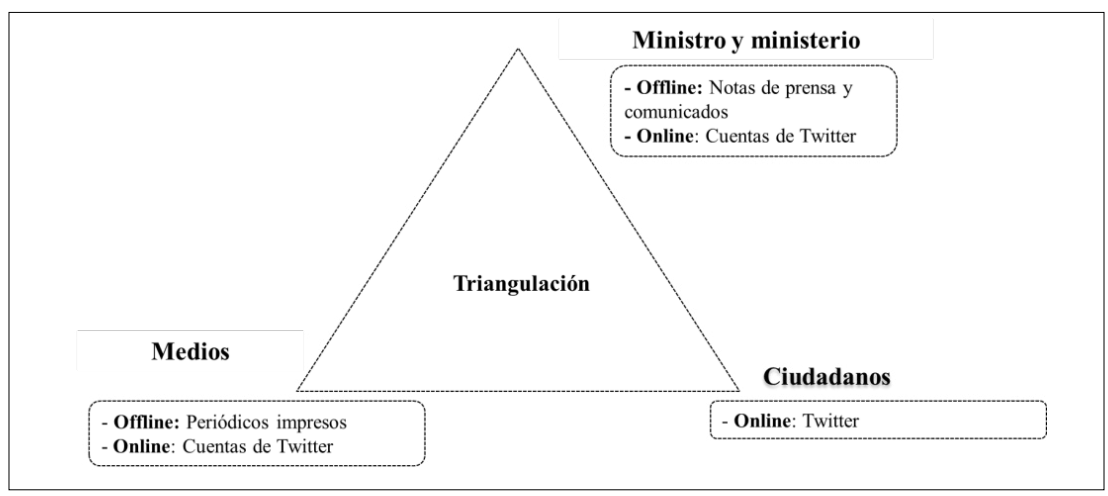

Fuente: elaboración propia

Para llevar a cabo esta investigación se ha realizado un análisis de contenido. De esta forma, se puede estudiar la comunicación en Twitter y en los medios tradicionales de forma cuantitativa y objetiva. Se ha diseñado una guía de análisis y un código para estudiar cada caso.

Para el análisis de las cuentas del ministro y ministerio en Twitter se han codificado las unidades de análisis de las cuentas de los Ministros de Educación, Cultura y Deporte José Ignacio Wert (@Jiwert) e Iñigo Méndez de Vigo (@IMendezdeVigo) y de la cuenta del Ministerio de Educación (@educaciongob). En cuanto a las fechas, se ha analizado el primer año desde el nombramiento de los dos ministros y un año del ministerio, que engloba a ambos ministros.

Las variables que se han seleccionado para este análisis son las siguientes:

1. Número del tweet: es el número que sirve para identificar la unidad de análisis.

2. Fecha: con formato $\mathrm{dd} / \mathrm{mm} / \mathrm{aa}$.

3. Importancia del tweet. si tiene imagen, video, enlaces, etc. 
4. Educación: si trata de educación o no.

5. Juzgado: aquella persona de la que se juzga algo; es decir, a la que se le atribuye un juicio.

6. Juzgador: aquel interlocutor (persona/institución/partido, etc.) que juzga algo sobre el ministro, ministerio o política pública.

7. Juicio cualitativo: el tweet que se haya publicado en la cuenta del ministro o ministerio.

8. Juicio cuantitativo: lo que el juzgador juzga al juzgado.

9. Tipo de legitimidad: personal, estructural, procedimental o consiguiente.

10. Sentido del juicio: si el atributo es positivo, negativo o neutro.

Respecto al análisis en discursos y comunicados se han codificado las unidades de análisis de las notas de prensa y comunicados publicadas en la página web del Ministerio de Educación, Cultura y Deporte. Solo se han codificado aquellas que hacen referencia a temas de educación o nombramientos. Las fechas analizadas son desde el 20 de diciembre de 2011 hasta el 31 de diciembre de 2015.

Las variables que se han tenido en cuenta para este análisis son las siguientes:

1. Número de nota de prensa o comunicado: es el número que sirve para identificar la unidad de análisis.

2. Fecha: con formato dd/mm/aa.

3. Juzgado: aquella persona de la que se juzga algo; es decir, a la que se le atribuye un juicio.

4. Juzgador: aquel interlocutor (persona/institución/partido, etc.) que juzga algo sobre alguien.

5. Juicio cualitativo: el juicio que se haya publicado en nota de prensa.

6. Juicio cuantitativo: lo que el juzgador juzga al juzgado.

7. Tipo de legitimidad: personal, estructural, procedimental o consiguiente.

8. Sentido del juicio: si el atributo es positivo, negativo o neutro.

Para el análisis en prensa en Twitter se han codificado las unidades de análisis de las cuentas de El País (@el_pais), El Mundo (@elmundoes) y ABC (@abc_es). Se han analizado los tweets sobre los ministros José Ignacio Wert e Iñigo Méndez de Vigo y sobre el Ministerio de Educación del 20 de diciembre de 2011 hasta el 20 de noviembre de 2015.

Las variables que se han seleccionado para este análisis son las siguientes:

1. Número del tweet: es el número que sirve para identificar la unidad de análisis.

2. Medio: El País, El Mundo y ABC.

3. Fecha: con formato $\mathrm{dd} / \mathrm{mm} / \mathrm{aa}$.

4. Importancia del tweet: si tiene imagen, video, enlaces, etc.

5. Educación: si se trata de educación o no.

6. Juzgado: aquella persona de la que se juzga algo; es decir, a la que se le atribuye un juicio.

7. Juzgador: aquel interlocutor (persona/institución/partido, etc.) que juzga algo sobre el ministro, ministerio o política pública. 
8. Juicio cualitativo: el tweet que se haya publicado en la cuenta de los medios.

9. Juicio cuantitativo: lo que el juzgador juzga al juzgado

10. Tipo de legitimidad: personal, estructural, procedimental o consiguiente.

11. Sentido del juicio: si el atributo es positivo, negativo o neutro.

En caso de la prensa impresa, se han codificado las unidades de análisis que incluyen en sus titulares, subtítulos, antetítulos o en los párrafos del texto de la noticia alguno de los siguientes términos: "Ministro de Educación, Cultura y Deporte", "José Ignacio Wert”, "Iñigo Méndez de Vigo" o "Ministerio de Educación, Cultura y Deporte. El análisis se ha realizado en diarios escogidos entre el 22 de diciembre de 2011 hasta el 20 de noviembre de 2015.

Para este análisis las variables que se han elegido son las siguientes:

1. Número de noticia: es el número que sirve para identificar la unidad de análisis.

2. Medio: El País, El Mundo y ABC.

3. Fecha: con formato dd/mm/aa.

4. Página: si la página es par, impar o doble página el número de página en la que se sitúa la noticia.

5. Extensión (incluyendo fotografías y gráficos): menos de un cuarto de página, menos de media página, media página o más, página completa o más de una página.

6. Juzgado: aquella persona de la que se juzga algo; es decir, a la que se le atribuye un juicio.

7. Juzgador: aquel interlocutor (persona/institución/partido, etc.) que juzga algo sobre el ministro, ministerio o política pública.

8. Juicio cualitativo: el juicio completo que aparezca en primer lugar.

9. Juicio cuantitativo: lo que el juzgador juzga al juzgado

10. Tipo de legitimidad: personal, estructural, procedimental o consiguiente.

11. Sentido del juicio: si el atributo es positivo, negativo o neutro.

Por último, para analizar los juicios de legitimidad de los ciudadanos se han seleccionado dos hashtags en Twitter. En concreto, se han codificado las unidades de análisis de las cuentas que han utilizado los hashtags \#JoseIgnacioWert y \#IñigoMéndezdeVigo. Se han analizado los tweets de dichos hashtags del 20 de diciembre de 2011 hasta el 31 de diciembre de 2015. Las variables que se han seleccionado para este análisis son las siguientes:

1. Número del tweet: es el número que sirve para identificar la unidad de análisis.

2. Que hashtag: \#joseignaciowert o \#IñigoMéndezDeVigo

3. Fecha: con formato $\mathrm{dd} / \mathrm{mm} / \mathrm{aa}$.

4. Importancia del tweet: si tiene imagen, video, enlaces, etc.

5. Educación: si trata de educación o no.

6. Juzgado: aquella persona de la que se juzga algo; es decir, a la que se le atribuye un juicio.

7. Juzgador: aquel interlocutor (persona/institución/partido, etc.) que juzga algo sobre el ministro, ministerio o política pública. 
8. Juicio cualitativo: el tweet que se haya publicado.

9. Juicio cuantitativo: lo que el juzgador juzga al juzgado.

10. Tipo de legitimidad: personal, estructural, procedimental o consiguiente.

11. Sentido del juicio: si el atributo es positivo, negativo o neutro.

Con esta investigación se quiere explorar el grado de congruencia entre los juicios de los distintos actores (organización, medios y ciudadanos). Teniendo en cuenta los datos de encuestas sobre la baja popularidad del ministro y del ministerio (que, aunque no se haya preguntado como tal, se pueden tomar como asociados a baja legitimidad y baja reputación), la hipótesis afirma que habrá poca congruencia. Pero, además, dado que los medios juegan un papel importante en la legitimidad (la legitimidad de la que gozan las organizaciones en sus públicos viene moderada por la legitimidad mediática), se formulan las siguientes cuestiones:

Pregunta de investigación 1: ¿Distan mucho los juicios de la organización con los que realizan sus públicos (medios y ciudadanos) sobre las mismas cuestiones?

Se ha diseñado un sistema de codificación para evaluar los tipos de juicios que se incluyen en los juicios emitidos. Esta variable recoge los siguientes atributos: confianza, transparencia, capacidad para justificar y explicar decisiones, consensuar, conforme a la ley, eficacia, responsabilidad, compromiso, simpatía, capacidad de comunicación, corrupción, cercanía, popularidad, trayectoria, ejemplaridad y liderazgo.

Pregunta de investigación 2: ¿Los juicios de los ciudadanos tienden a asemejarse más a los juicios de los medios que a los de la organización en cuanto al tipo de legitimidad y al sentido del juicio?

A continuación, se analizan los resultados obtenidos en esta investigación.

\section{Resultados}

En este apartado se muestran los resultados de los datos obtenidos una vez realizado el análisis de contenido en los tres puntos de observación. Para aclarar la explicación, cuando se tratan los datos de los actores se exponen de dos maneras. Por un lado, hablamos de "actores sin acumular", es decir, cuando se suman todos los datos de un mismo punto de observación y diferenciando entre el contexto online y offline. Y, por otro lado, mencionamos los "actores acumulados", que hacen referencia a los datos por separado.

\subsection{La congruencia en el contenido de la organización versus los medios y los ciudadanos.}

Con la variable "Juicio", se quiso obtener información un poco más precisa sobre cuáles son los rasgos o características a los que se refieren los juicios de legitimidad en cada actor. 
Tabla 1. El contenido de los juicios de los actores sin acumular

\begin{tabular}{|c|c|c|c|c|c|c|c|}
\hline \multirow[b]{2}{*}{ Juicio } & \multicolumn{3}{|c|}{ Organización online } & \multirow{2}{*}{$\begin{array}{c}\text { Organización offline } \\
\text { Notas de prensa y } \\
\text { comunicados }\end{array}$} & \multirow{2}{*}{$\begin{array}{l}\text { Prensa online } \\
\text { Prensta Twitter }\end{array}$} & \multirow{2}{*}{$\begin{array}{l}\text { Prensa offline } \\
\text { Prensa imprensa }\end{array}$} & \multirow{2}{*}{\begin{tabular}{|c|} 
Ciudadanos \\
Tweets ciudadanos
\end{tabular}} \\
\hline & Twitter Wert & $\begin{array}{c}\text { Twitter Méndez de } \\
\text { Vigo }\end{array}$ & $\begin{array}{l}\text { Twitter Ministerio } \\
\text { Educación }\end{array}$ & & & & \\
\hline Confianza & $0 \%(0)$ & $0,6 \%(1)$ & $0 \%(0)$ & $0.8 \%(3)$ & $0 \%(0)$ & $0 \%(0)$ & $0 \%(0)$ \\
\hline Transparencia & $0 \%(0)$ & $0 \%(0)$ & $0,8 \%(15)$ & $1,3 \%(5)$ & $0 \%(0)$ & $0 \%(0)$ & $0,6 \%(2)$ \\
\hline Justificar y explicar & $14,7 \%(5)$ & $6,6 \%(12)$ & $0,7 \%(14)$ & $17,4 \%(69)$ & $22,8 \%(28)$ & $15 \%(40)$ & $18,2 \%(58)$ \\
\hline Consensuar & $5,9 \%(2)$ & $17,7 \%(32)$ & $37 \%(720)$ & $22,2 \%(96)$ & $11,4 \%(14)$ & $19,5 \%(52)$ & $20,7 \%(66)$ \\
\hline Conforme a ley & $0 \%(0)$ & $0 \%(0)$ & $0,1 \%(1)$ & $1,8 \%(7)$ & $0,8 \%(1)$ & $4,5 \%(12)$ & $0,6 \%(2)$ \\
\hline Eficacia & $47,1 \%(16)$ & $34,8 \%(63)$ & $61,1 \%(1.187)$ & $53,7 \%(213)$ & $60,2 \%(74)$ & $50,9 \%(136)$ & $48 \%(153)$ \\
\hline Responsabilidad & $0 \%(0)$ & $0 \%(0)$ & $0,2 \%(3)$ & $0 \%(0)$ & $0 \%(0)$ & $0,4 \%(1)$ & $0 \%(0)$ \\
\hline Compromiso & $0 \%(0)$ & $0 \%(0)$ & $0,1 \%(2)$ & $0,3 \%(1)$ & $0 \%(0)$ & $0,7 \%(2)$ & $0,6 \%(2)$ \\
\hline Simpatía & $5,9 \%(2)$ & $1,1 \%(2)$ & $0 \%(0)$ & $0 \%(0)$ & $0,8 \%(1)$ & $3 \%(8)$ & $5,3 \%(17)$ \\
\hline $\begin{array}{l}\text { Capacidad de } \\
\text { comunicación }\end{array}$ & $0 \%(0)$ & $0 \%(0)$ & $0 \%(0)$ & $0 \%(0)$ & $0 \%(0)$ & $0,4 \%(1)$ & $0 \%(0)$ \\
\hline Corrupción & $0 \%(0)$ & $0 \%(0)$ & $0 \%(0)$ & $0 \%(0)$ & $0 \%(0)$ & $0,4 \%(1)$ & $1,6 \%(5)$ \\
\hline Cercanía & $23,5 \%(8)$ & $39,2 \%(71)$ & $0 \%(0)$ & $0,3 \%(1)$ & $0 \%(0)$ & $0 \%(0)$ & $0,3 \%(1)$ \\
\hline Popularidad & $0 \%(0)$ & $0 \%(0)$ & $0,1 \%(1)$ & $0 \%(0)$ & $1,6 \%(2)$ & $1,5 \%(4)$ & $0 \%(0)$ \\
\hline Trayectoria & $2,9 \%(1)$ & $0 \%(0)$ & $0,1 \%(1)$ & $0 \%(0)$ & $1,6 \%(2)$ & $3,7 \%(10)$ & $4,1 \%(13)$ \\
\hline Ejemplaridad & $0 \%(0)$ & $0 \%(0)$ & $0 \%(0)$ & $0,5 \%(2)$ & $0 \%(0)$ & $0 \%(0)$ & $0 \%(0)$ \\
\hline Liderazgo & $0 \%(0)$ & $0 \%(0)$ & $0 \%(0)$ & $0 \%(0)$ & $0,8 \%(1)$ & $0 \%(0)$ & $0 \%(0)$ \\
\hline $\begin{array}{l}\mathrm{N}=3.265 \\
\text { Chi-cuadrado }=0,000\end{array}$ & & & & & & & \\
\hline
\end{tabular}

Tal y como se muestra en la Tabla 1, el atributo predominante en todos los actores sin acumular es el de "eficacia", a excepción del ministro Méndez de Vigo en Twitter, que en sus juicios utiliza más el atributo "cercanía o lejanía".

Entre los diferentes actores de la organización existen contrastes. Los datos muestran que para Wert el segundo atributo más empleado fue "cercanía o lejanía" y para Méndez de Vigo "eficacia". Sin embargo, para el Ministerio en Twitter o para las notas de prensa emitidas por éste, fue "la capacidad para interactuar o consensuar" el atributo más relevante.

En la prensa también hay diferencias: mientras que la prensa impresa tiene más congruencia con la organización en Twitter y con las notas de prensa con el atributo "capacidad para interactuar o consensuar" (19,5\%), como el segundo más empleado en los juicios, la prensa en Twitter le da más importancia al de "disposición para justificar y explicar decisiones" $(22,8 \%)$.

Sin embargo, los ciudadanos dieron más relevancia en sus juicios, después de la "eficacia", al atributo "conforme a la ley" $(20,7 \%)$.

A mayores efectos de congruencia, se realizó un análisis de correlaciones bivariadas entre la jerarquización del contenido de los juicios de cada actor especifico (sin acumular), es decir, a partir de ordenar de mayor a menor, según el porcentaje de frecuencia obtenido en cada caso para comprobar la similitud entre los diferentes rankings. Los resultados se exponen en la Tabla 2. 
Tabla 2. Correlaciones bivariadas entre los atributos de los distintos actores sin acumular

\begin{tabular}{|c|c|c|c|c|c|c|c|}
\hline & Twitter Wert & $\begin{array}{c}\text { Twitter Méndez } \\
\text { de Vigo }\end{array}$ & $\begin{array}{c}\text { Twitter Ministerio } \\
\text { Educación }\end{array}$ & $\begin{array}{l}\text { Notas de prensa } \\
\text { y comunicados }\end{array}$ & $\begin{array}{l}\text { Prensa } \\
\text { Twitter }\end{array}$ & $\begin{array}{l}\text { Prensa } \\
\text { impresa }\end{array}$ & $\begin{array}{l}\text { Tweets } \\
\text { ciudadanos }\end{array}$ \\
\hline Twitter Wert & 1 & & & & & & \\
\hline Twitter Méndez de Vigo & $.561 *$ & 1 & & & & & \\
\hline $\begin{array}{c}\text { Twitter Ministerio } \\
\text { Educación }\end{array}$ & - & -.329 & 1 & & & & \\
\hline $\begin{array}{l}\text { Notas de prensa y } \\
\text { comunicados }\end{array}$ & - & .302 & - & 1 & & & \\
\hline Prensa Twitter & .403 & - & - & $-.554 *$ & 1 & & \\
\hline Prensa impresa & - & - & .357 & -.307 & - & 1 & \\
\hline Tweets ciudadanos & .428 & - & - & .403 & - & - & 1 \\
\hline $\mathrm{N}=16$ & & & & & & & \\
\hline
\end{tabular}

Fuente: elaboración propia

Los datos muestran que hay correlación estadísticamente significativa (Rho de Spearman $=.02$ ) entre los atributos del juicio en Twitter que fueron más importantes para el ministro Wert y aquellos que lo fueron para el ministro Méndez de Vigo (coeficiente .56). Es éste, por otra parte, un resultado esperado, pues, aunque sean ministros diferentes, son del mismo gobierno y del mismo partido y, por tanto, es lógico que su discurso guarde una cierta congruencia. Se produce lo que Chadwick llama "híbridos organizacionales" (2007), concepto con el que se afirma que los líderes, además de utilizar los medios tradicionales, han adoptado el uso de los medios sociales para enriquecer la comunicación que se ofrece al ciudadano; y, en este caso, se observa que hay consistencia entre el mensaje del ministro Méndez de Vigo con el mensaje del ministro Wert.

Los datos arrojan otra relación estadísticamente significativa (Rho de Spearman $=.02$ ), pero interesante, en este caso es negativa (coeficiente -.55), entre los atributos de los juicios formulados por la organización en las notas de prensa y aquellos formulados por la prensa en Twitter. Es decir, parece producirse un alejamiento entre la comunicación de la organización offline y la comunicación en la prensa online, por el momento parece constatarse que, por lo que se refiere al contenido del juicio, la organización no logra determinar con sus notas de prensa la agenda de temas de la prensa online. Y puede haber pasado lo contrario de lo que sugiere Giansante (2015): la organización tiene que tener en cuenta las diferencias que existen entre los medios tradicionales y los sociales. Es decir, si una organización manda una nota de prensa a los medios de comunicación, tiene que pensar también en otra estrategia para los medios sociales. No parece que tal haya sido el caso, según estos datos.

Aunque sin significación estadística, también cabe destacar que hubo correlación positiva entre el contenido de los juicios emitidos por Wert, por un lado, con los de la prensa en Twitter (.403) y, por otro, con los tweets de los ciudadanos (.428), correlación que se explica por la naturaleza del medio: la comunicación online puede estar inclinando a los distintos actores hacia un mismo tipo de atributos. 
También sin significación estadística, hay cierta correlación entre la comunicación del Ministerio en Twitter y la prensa impresa (.357), y entre los atributos de las notas de prensa y los de los ciudadanos (.403). Sin embargo, y sorprendentemente, hay correlación negativa en el contenido de los juicios del ministro Méndez de Vigo y el Ministerio de Educación en Twitter (-.329), como si éste hubiera llevado una agenda propia de atributos independiente de la organización a la que representa. Por último, resulta interesante comprobar una correlación también negativa (-.307) entre las notas de prensa de la organización y el contenido de los juicios de la prensa, ya sea impresa u online. Este es el segundo dato relevante al que llegamos que muestra que las notas de prensa del Ministerio no han sido capaces de influir en la prensa.

En respuesta a la pregunta de investigación 1, con estos datos, podemos afirmar que distan mucho los juicios formulados por la organización con los de la prensa y los de los ciudadanos. A pesar de que el atributo más usado en todos los juicios es el de eficacia en todos los actores, salvo por el ministro Méndez de Vigo, no existe correlación entre los tres puntos de observación. Además, en el caso de las notas de prensa y la prensa online, se da de forma negativa, es decir, a los valores altos de una le corresponden valores bajos de la otra o viceversa. En definitiva, parece ponerse de manifiesto que aquello que es central para construir la legitimidad de una organización, a saber, la congruencia entre sus juicios y los de sus distintos stakeholders, no se ha producido en lo que se refiere el Ministerio de Educación en la etapa estudiada en este trabajo.

\subsection{La congruencia en el tipo legitimidad de la organización versus los medios y los ciudadanos}

Para analizar la congruencia en el tipo de legitimidad de la organización frente a los otros dos puntos de observación, nos vamos a basar en los datos que muestran la Tabla 3 y 4.

Tabla 3. Las cuatro dimensiones de legitimidad de los actores sin acumular

\begin{tabular}{|c|c|c|c|c|c|}
\hline \multirow{2}{*}{\multicolumn{2}{|c|}{ Actores }} & \multicolumn{4}{|c|}{ Tipo de legitimidad } \\
\hline & & Procedimental & Consiguiente & Estructural & Personal \\
\hline \multirow{3}{*}{ Organización online } & Twitter Wert & $70 \%(21)$ & $10 \%(3)$ & $0 \%(0)$ & $20 \%(6)$ \\
\hline & Twitter Méndez de Vigo & $78,8 \%(82)$ & $15,4 \%(16)$ & $0 \%(0)$ & $5,8 \%(6)$ \\
\hline & Twitter Ministerio Educación & $95 \%(1.847)$ & $1,5 \%(30)$ & $3,4 \%(67)$ & $0 \%(0)$ \\
\hline Organización offline & Notas de prensa y comunicados & $91,4 \%(363)$ & $8,6 \%(34)$ & $0 \%(0)$ & $0 \%(0)$ \\
\hline Prensa online & Prensa Twitter & $79,8 \%(99)$ & $9,7 \%(12)$ & $0 \%(0)$ & $10,5 \%(13)$ \\
\hline Prensa offline & Prensa impresa & $51,3 \%(139)$ & $35,8 \%(97)$ & $0,4 \%(1)$ & $12,5 \%(34)$ \\
\hline Ciudadanos & Tweets ciudadanos & $74,8 \%(243)$ & $6,5 \%(21)$ & $0 \%(0)$ & $18,8 \%(61)$ \\
\hline \multicolumn{6}{|l|}{$\mathrm{N}=3.195$} \\
\hline \multicolumn{6}{|l|}{ Chi-cuadrado $=0,000$} \\
\hline
\end{tabular}

Fuente: elaboración propia 
Tabla 4. Las cuatro dimensiones de legitimidad de los actores acumulados

\begin{tabular}{|c|c|c|c|c|}
\hline \multirow{2}{*}{ Actores } & \multicolumn{4}{|c|}{ Tipo de legitimidad } \\
\cline { 2 - 4 } & Procedimental & Consiguiente & Estructural & Personal \\
\hline Organización online & $81,3 \%(1.950)$ & $9 \%(49)$ & $1,1 \%(67)$ & $8,6 \%(12)$ \\
\hline Organización offline & $91,4 \%(363)$ & $8,6 \%(34)$ & $0 \%(0)$ & $0 \%(0)$ \\
\hline Prensa online & $79,8 \%(99)$ & $9,7 \%(12)$ & $0 \%(0)$ & $10,5 \%(13)$ \\
\hline Prensa offline & $51,3 \%(139)$ & $35,8 \%(97)$ & $0,4 \%(1)$ & $12,5 \%(34)$ \\
\hline Ciudadanos & $74,8 \%(243)$ & $6,5 \%(21)$ & $0 \%(0)$ & $18,8 \%(61)$ \\
\hline N=3.195 & \multicolumn{4}{|l|}{} \\
\hline Chi-cuadrado $=0,000$
\end{tabular}

Fuente: elaboración propia

Los datos muestran que, efectivamente, no hay congruencia entre los distintos actores, es decir, hay preocupación por diferentes cuestiones en los tres niveles observados. Y los ciudadanos están más en línea con la prensa que con la organización; pero curiosamente, no con la prensa offline sino con la online: los patrones de preocupación por los resultados y el líder que reflejan los ciudadanos coinciden con los de la prensa online. La prensa offline es la que lidera el discurso por los resultados de gestión, pero no le siguen los ciudadanos. Y, en respuesta a la pregunta de investigación 1, medios y ciudadanos no concuerdan (no siguen) el mensaje que formuló la organización: ninguno comparte esa casi exclusiva preocupación por los procedimientos.

\subsection{La congruencia en el sentido del juicio de la organización versus los medios y los ciudadanos.}

La Tabla 5 muestra el sentido de los juicios de los actores acumulados.

Tabla 5. El sentido del juicio de los actores acumulados

\begin{tabular}{|c|c|c|c|}
\hline \multirow{2}{*}{ Actores } & \multicolumn{3}{|c|}{ Sentido del juicio } \\
\cline { 2 - 4 } & Positivo & Negativo & Neutro \\
\hline Organización online & $17,9 \%(99)$ & $0 \%(0)$ & $82,1 \%(2.079)$ \\
\hline Organización offline & $9,1 \%(36)$ & $0 \%(0)$ & $90,9 \%(361)$ \\
\hline Prensa online & $7,3 \%(9)$ & $42,7 \%(53)$ & $50 \%(62)$ \\
\hline Prensa offline & $16,1 \%(44)$ & $51,8 \%(142)$ & $32,1 \%(88)$ \\
\hline Ciudadanos & $8,8 \%(29)$ & $52,3 \%(172)$ & $38,9 \%(128)$ \\
\hline N=3.302 & \\
\hline Chi-cuadrado $=0,000$
\end{tabular}

Fuente: elaboración propia 
Los datos arrojan una gran disparidad entre los juicios de los tres actores: la elevada neutralidad de la organización no es seguida ni por los medios ni por los ciudadanos. Estos, como era de esperar, son más negativos (52,3\%) que la organización (0), y con su negatividad se acercan más a los juicios de los medios. Pero, curiosamente, hay más acercamiento de los juicios de los ciudadanos a la prensa offline (con negatividad de 51,8\%) que a la online (con negatividad del 42,7). Decimos "curiosamente", pues podría esperarse que, al ser online la comunicación del ciudadano que se analiza, sería a la prensa online a la que se parecen sus juicios; $y$ tal no es el caso.

Por el interés que tienen los datos, reproducimos en la Tabla 6 los actores sin acumular.

Tabla 6. El sentido del juicio de los actores sin acumular

\begin{tabular}{|c|c|c|c|c|}
\hline & \multirow{2}{*}{ Actores } & \multicolumn{3}{|c|}{ Sentido del juicio } \\
\hline & & Positivo & Negativo & Neutro \\
\hline \multirow{3}{*}{$\begin{array}{c}\text { Organización } \\
\text { online }\end{array}$} & Twitter Wert & $15,2 \%(7)$ & $0 \%(0)$ & $84,8 \%(39)$ \\
\hline & Twitter Méndez de Vigo & $37,4 \%(70)$ & $0 \%(0)$ & $62,6 \%(117)$ \\
\hline & Twitter Ministerio Educación & $1,1 \%(22)$ & $0 \%(0)$ & $98,9 \%(1.923)$ \\
\hline $\begin{array}{c}\text { Organización } \\
\text { offline }\end{array}$ & Notas de prensa y comunicados & $9,1 \%(36)$ & $0 \%(0)$ & $90,9 \%(361)$ \\
\hline Prensa online & Prensa Twitter & $7,3 \%(9)$ & $42,7 \%(53)$ & $50 \%(62)$ \\
\hline Prensa offline & Prensa impresa & $16,1 \%(44)$ & $51,8 \%(142)$ & $32,1 \%(88)$ \\
\hline Ciudadanos & Tweets ciudadanos & $8,8 \%(29)$ & $52,3 \%(172)$ & $38,9 \%(128)$ \\
\hline \multicolumn{5}{|c|}{$\mathrm{N}=3.302$} \\
\hline \multicolumn{5}{|c|}{ Chi-cuadrado $=0,000$} \\
\hline
\end{tabular}

Fuente: elaboración propia

Los datos muestran que es la comunicación online del Ministerio la que incrementa la neutralidad de la organización, pues cuando se ven los datos por separado, se observa que los líderes, aunque se comunican con un discurso predominantemente neutro, introducen más afirmaciones positivas que la organización en su perfil. Hay una clara diferencia entre la comunicación de Wert y la comunicación de su sucesor Méndez de Vigo, que casi dobla las afirmaciones positivas de aquél.

Por otro lado, en la tabla se observan diferencias entre la prensa impresa y en Twitter. Mientras la primera es más negativa $(51,8 \%)$, la segunda es neutral (50\%) y negativa $(42,7 \%)$. En los ciudadanos predomina el sentido negativo (52,3\%). Por tanto, en cuanto al sentido del juicio, tienen mayor congruencia con la prensa impresa que con el resto de los actores.

El análisis del contraste muestra claramente que hay una disparidad entre la organización y medios y ciudadanos, y esta disparidad se produce en todos los formatos de la comunicación de la organización (las cuentas de los ministros y la del ministerio en Twitter, pero también en las notas de prensa y comunicados). La prensa en Twitter sigue un poco más las pautas organizacionales que la prensa impresa, que es la que muestra más similitud con el discurso de los ciudadanos.

Una vez analizados los datos, podemos responder a la pregunta de investigación 2: los juicios de los ciudadanos tienden a asemejarse más a los de los medios que a los de la organización en cuanto al tipo de legitimidad y al sentido del juicio. 
Aunque hay que matizar que, en cuanto al tipo de legitimidad, aunque predominen en los dos la legitimidad procedimental, los ciudadanos introducen, como los medios, otro tipo de preocupaciones. En cuanto al sentido del juicio, los juicios de los ciudadanos se asemejan más a la prensa que a los diferentes actores de la organización. Pero esa semejanza se produce más con la prensa impresa que con la online, un resultado que consideramos de interés.

\section{Conclusiones}

Tras el análisis de los datos, las conclusiones que hemos alcanzado en el presente trabajo son las siguientes.

Concluimos que los juicios formulados por la organización distan mucho con los de la prensa y los de los ciudadanos, en respuesta a la pregunta de investigación 1. A pesar de que el atributo más usado por todos los actores en sus juicios es el de "eficacia", no existe correlación entre los tres puntos de observación (organización, medios y ciudadanos). Los datos parecen haber mostrado una falta de congruencia dentro de la propia organización; y quizá sea ésta la que se proyecta hacia los medios (incluso la comunicación de la organización a través de notas de prensa se correlaciona negativamente con la prensa online) y a los ciudadanos.

En cuanto al tipo de legitimidad, los resultados muestran que la predominante, como se ha dicho, es la legitimidad procedimental. Sin embargo, mientras que en la organización existe un predominio total, en la prensa la procedimental estuvo menos presente que la consiguiente, y en los ciudadanos la más significativa fue la personal.

Una vez analizados los datos podemos afirmar que los juicios de los ciudadanos tienden a asemejarse más a los de los medios más que a los de la organización en cuanto al tipo de legitimidad y al sentido del juicio, en respuesta a la pregunta de investigación 2.

Aunque hay que matizar que, en cuanto al tipo de legitimidad, predomina en los dos actores la legitimidad procedimental, si bien los ciudadanos y los medios introducen otro tipo de preocupaciones. En cuanto al sentido del juicio, los juicios de los ciudadanos se asemejan más a la prensa que a los diferentes actores de la organización. Pero esa semejanza se produce más con la prensa impresa que con la online. Por tanto, en líneas generales se puede concluir que se cumple lo que dice Chadwick (2013), es decir, son los medios tradicionales los que influyen más que los medios online en los ciudadanos.

Tras esta discusión, parece ponerse de manifiesto que aquello que es central para construir la legitimidad de una organización, a saber, la congruencia entre sus juicios y los de sus distintos stakeholders, no se ha producido en lo que se refiere el Ministerio de Educación en la etapa estudiada en este trabajo.

Siguiendo el enfoque de legitimidad mediática de Robles y Rodríguez (2017), concluimos que las instituciones públicas deben vigilar el eco mediático a la hora de elaborar sus estrategias comunicativas para cuidar su legitimidad.

En definitiva, los datos muestran la necesidad de elaborar estrategias de comunicación que combinen los resultados y los aspectos personales con los procesos para no distanciarse de los medios y los ciudadanos. Además, estas estrategias de comunicación deberían adaptarse a los diferentes tipos de medios.

A partir de aquí se abren líneas de investigación sobre la dinámica de los juicios de legitimidad en la Administración Pública. Por otro lado, queda pendiente el análisis de la comunicación de los ciudadanos de forma offline, a través de 
encuestas ad-hoc y específicas sobre legitimidad, con el fin de poder comparar los tres puntos de observación desde una perspectiva offline. Otra importante futura línea de investigación sería la de profundizar en la legitimidad personal del líder, es decir, analizar la importancia que tienen los atributos sobre los aspectos personales del líder político dentro de la identidad del mismo.

Por último, y por lo que respecta a la investigación en el campo de los intangibles en el sector público, esta investigación se ha centrado en el bien intangible legitimidad, por lo que sería conveniente ampliar el estudio con otros bienes como el capital intelectual o el capital social, con el propósito de ver, en último término, como la construcción de intangibles contribuye a la recuperación de la confianza en las organizaciones públicas.

\section{Referencias bibliográficas}

Baamonde, X. M. (2011). Las redes sociales como herramientas de Relaciones Públicas de las instituciones europeas. Correspondencias \& Análisis, (1), 67-82.

Bennett, W. L., \& Segerberg, A. (2014). La comunicación en los movimientos. De los medios de comunicación de masas a las redes sociales. Telos: Cuadernos de comunicación e innovación, (98), 58-70.

Bertot, J. C.; Jaeger, P. T.; and Grimes, J. M. (2010). Using ICTs to create a culture of transparency: E-government and social media as openness and anti-corruption tools for societies. Government Information Quarterly, 27 (3), 264-271. DOI http://dx.doi.org/10.1016/j.giq.2010.03.001.

Bitektine, A. (2011). Toward a theory of social judgments of organizations: The case of legitimacy, reputation, and status. Academy of Management Review, 36 (1), 151-179.

Bossi, A., Fuentes, Y. y Serrano, C. (2005). Reflexiones en torno a la aplicación del capital intelectual en el sector público. Revista Española de Financiación y Contabilidad, 34 (124), 211-245.

Canel, M.J. (2015). La comunicación de las instituciones públicas para la regeneración democrática. En F. Llera (Dir.), La regeneración democrática en España. Madrid: Centro de Estudios Constitucionales.

Canel, M. J., y García- Molero, Á. (2013). Comunicar gobiernos fiables. Análisis de la confianza como valor intangible del Gobierno de España. Zer-Revista de Estudios de Comunicación, 18(34).

Canel, M. J. \& Luoma-Aho, V. (2019). Public Sector Communication. Closing Gaps Between Citizens and Organizations. Boston: Wiley-Blackwell.

Castelló, I., Etter, M. y Arup Nielsen, F. (2016). Strategies of Legitimacy Through Social Media: The Networked Strategy. Journal of Management Studies. Vol 53, n. 3, pp. 402-432.

Catalina García, B.; López De Ayala López, Mª C.; y Fernández Fernández, J. G. (2015). Twitter como plataforma de los alcaldes para la comunicación pública. Estudios sobre el Mensaje Periodístico, 21 (2), 757-772. DOI http://dx.doi. org/10.5209/rev_ESMP.2015.v21.n2.50884.

Chadwick, A. (2007). Digital network repertoires and organizational hybridity. Political Communication, 24(3), 283-301. 
Chadwick, A. (2013). The hybrid media system: politics and power. New York: Oxford University Press.

Chen, Y., Caramanis, C., \& Mannor, S. (2013). Robust sparse regression under adversarial corruption. In International Conference on Machine Learning (pp. 774-782).

Chun Soon A.; Shulman, S.; Sandoval, R.; and Hovy, E. (2010). Government 2.0: making connections between citizens, data and government. Information Polity, 15 (1-2), 1-9. DOI: https://doi.org/10.3233/IP-2010-0205.

Colleoni, E. (2013). CSR communication strategies for organizational legitimacy in social media. Corporate Communications: an international journal,18(2), 228-248.

Congosto, M. L. y Aragón, P. (2012). Twitter, del sondeo a la sonda: nuevos canales de opinión, nuevos métodos de análisis. Revista Más Poder Local, 12, 50-56.

De Ramón Carrión, M. (2014). Presencia de la información especializada en defensa en la red social Twitter. Revista de Comunicación de la SEECI, 34, 95-112. DOI http://dx.doi.org/10.15198/seeci.2014.34.95-112.

Deephouse, D. y Carter, S. (2005). An Examination of Differences Between Organizational Legitimacy and Organizational Reputation. Journal of Management Studies, 42(2), 329-360.

Díez Martín, F, Blanco González, A., y Prado Román, C. (2010). Legitimidad como factor clave del éxito organizativo. Investigaciones Europeas de Dirección y Economía de la Empresa, 16(3), 127-143.

DiMaggio, P., y Powell, W. W. (1983). The iron cage revisited: Collective rationality and institutional isomorphism in organizational fields. American Sociological Review, 48(2), 147-160.

García Carballo, Carlos (2012). La personalización de los mensajes en Twitter: caso del Ministerio de Educación y Ministerio de Cultura. Razón y palabra, 17 (79): http://www.redalyc.org/comocitar.oa?id=199524411067 Acceso en: 7 de abril de 2020.

Giansante, G. (2015). La comunicación política online: cómo utilizar la web para construir consenso y estimular la participación. Barcelona, España: Editorial UOC.

Grimmelikhuijsen, S. y Meijer, A. (2015). Does Twitter Increase Perceived Police Legitimacy? Public Administration Review, Vol. 75, Iss.4, 598-607

Jenkins, H. (2006). Convergence culture: Where old and new media collide. NYU press.

Luoma-aho, V. (2007). Neutral Reputation and Public Sector Organizations. Corporate Reputation Review, 10,124-143.

Manfredi Sánchez, J. L. y Femenía Guardiola, C. (eds., 2016). La diplomacia española ante el reto digital. Madrid, Ministerio de Asuntos Exteriores.

Martínez Rolán, X. y Piñeiro Otero, T. (2014). Del perfil electoral a la interacción con la ciudadanía: la apropiación de Twitter por los agentes del Parlamento de Galicia. Revista de la Asociación Española de Investigación de la Comunicación, 1 (2), 103- 115.

Pardo Baldeón, R. S. (2014). Análisis sobre el uso de Twitter en las administraciones locales de la provincia de Castellón. Miguel Hernández Communication Journal, 5, 361-379. 
Pollock, T. G. y Rindova, V. P. (2003). Media legitimation effects in the market for initial public offerings. Academy of Management Journal, 46(5), 631-642.

Ramos del Cano, F. (2013). Redes sociales en el entorno radiofónico: el uso de Twitter como fuente periodística en la Cadena SER. Revista Mediterránea de Comunicación: Mediterranean Journal of Communication, 4(2), 173-188.

Robles- López, C. M. (2017). La reputación y la legitimidad como bienes intangibles en el sector público. El caso del Ministro y el Ministerio de Educación, Cultura y Deporte (2011-2015). (Tesis Doctoral). Universidad Complutense de Madrid, Madrid, España. Disponible en: https://eprints.ucm.es/44275/1/T39126.pdf Acceso en: 11 marzo 2020.

Robles- López, C. M. (2019). La reputación y la legitimidad como bienes intangibles en el sector públic: el caso del ministro y el ministerio de educación, cultura y deporte (2011-2015). Mc Graw Hill.

Robles López, C. M. \& Canel Crespo, M. J. (2017). Exploring the dynamics of the legitimacy judgment about the public sector: the case of the Spanish Ministry of Education and its media legitimacy (2011-2015). Communication \& Society 30(3), 215-228.

Robles, C. M.; Rodríguez, C. (2017). El bien intangible legitimidad. En: Canel, M. J.; Piqueiras, P.; Ortega, G. (ed.). $L a$ comunicación de la Administración Pública: conceptos y casos prácticos de bienes intangibles. Madrid: Innap Investiga, 51-74.

Romero Portillo, J. y Mena Fernández, M. (2013). Parlamentos en Twitter: análisis de los contenidos y la interactividad en @Congreso_Es y @HouseofCommons. Comunicaçao Midiática, 8 (2), 232-259.

Sánchez, M. (2008). Papel de los intangibles y el capital intelectual en la creación y difusión del conocimiento en las organizaciones. Situación actual y retos de futuro. Arbor, 184(732), 575-594.

Simelio Solà, N. y Molina Rodríguez-Navas, P. (2014). Comunicación pública y participación ciudadana: el uso de Twitter en los ayuntamientos de Cataluña. Historia y Comunicación Social, 19 (especial febrero), 479-490. DOI http://dx.doi. org/10.5209/rev_HICS.2014.v19.45043

Suchman, M. (1995). Managing legitimacy: Strategic and institutional approaches. Academy of management review, 20(3), 571-610.

Thomas, J. C. (2013). Citizen, customer, partner: Rethinking the place of the public in public management. Public Administration Review, 73(6), 786-796.

Uslaner, E. M. (2010). A Culture of Corruption: Everyday Deception and Popular Discontent in Nigeria. By Daniel Jordan Smith. Perspectives on Politics, 8(4), 1175-1176.

Vázquez Sande, P. (2013). Alcaldes españoles en Twitter: ¿diálogo o monólogo?. Fonseca Journal of Communication, 7, 43-71. 